\title{
GB virus C
}

National Cancer Institute

\section{Source}

National Cancer Institute. GB virus C. NCI Thesaurus. Code C14328.

A species of enveloped viruses in the Flaviviridae family and Pegivirus genus. The genome is composed of linear, single-stranded, positive-sense RNA. GB Virus C has been shown to infect human cells in vitro and in vivo but has not yet been shown to be the causative agent of disease. 